\section{Military Technical College Kobry El-kobbah, Cairo, Egypt}

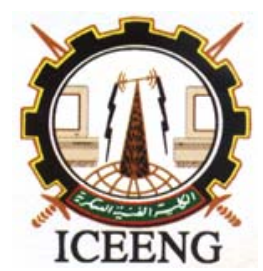

\author{
$5^{\text {th }}$ International Conference \\ on Electrical Engineering \\ ICEENG 2006
}

\title{
AUTONOMOUS UNDERWATER VEHICLE ROBUST CONTROL
}

\author{
G.A. Elnashar
}

Egyptian Armed Forces

\begin{abstract}
This paper discusses the autonomous underwater vehicle (AUV) control performance under uncertainty using two different methods, linear quadratic (LQ) servo with command following and sliding mode control (SMC). In spite of the uncertainty in our evaluations of the hydrodynamic forces, it is fortunate that the use of feedback control is able to compensate for this general lack of knowledge and to provide commands to actuators that control and stabilize the motion of underwater vehicles. Robustness is obtained by using feedback of key motion variables (wind, waves, and current) as measured by sensors to drive actuators which, in turn, manipulate the vehicle's motion so that changes in the behavior of the vehicle can be automatically compensated. In order to successfully recover or launch a vehicle it will be preferred for the vehicle to have the capability to compensate for this motion. This paper attempts to investigate a means by which a vehicle may be made to track, in depth, the dynamic motion for launch and recover at some significant depth below the surface. Design techniques for robust controllers typically use frequency response or state space techniques to specify control gains and even include observers and model based compensators to replace missing sensors with virtual sensors. While these techniques have definable robustness properties, sliding mode control and (LQ) servo with command following - techniques that can compensate for known nonlinear behavior - are convenient and has equally definable robustness properties.

This paper conducts robust control using (LQ) servo with command following and sliding mode control (SMC) which have been found useful and convenient in dealing with the uncertainty and general nonlinear nature of the models developed previously.
\end{abstract}

Keywords: Autonomous underwater vehicle, Robust Control, LQ servo command control, Sliding Mode Control.

\section{INTRODUCTION}

Conventional controllers design based on linear theory starts with the assumption that the 6 DOF underwater vehicle equation of motion can be described as a linear model linearized around a point of equilibrium. Stability of an underwater vehicle can be defined as the ability of returning an equilibrium state of motion after a disturbance without any corrective action, such as use of thruster power or control surfaces. One particular area of interest is the capability to deploy and recover AUV's from a tethered cage at some depth below the surface of the water. The challenge arrives upon recovery of the AUV to the cage platform. The cage platform has dynamics associated with it which are induced by wave motion effects of the ship to which the cage is tethered. In order to successfully recover a 
vehicle into a cage it will be necessary for the vehicle to have the capability to compensate for this motion when making its final approach to the cage.

Previous work in the field of AUV control has shown many different techniques for controlling an AUV's trajectory. From simple state feedback methods, to optimal methods like Linear Quadratic Regulator (LQR), to Lyapanov based methods like Sliding Mode Control (SMC), there are many methods which can be successfully utilized, each having particular advantages and disadvantages. Control engineers have studied autopilot performance from both classical and $H_{\infty}$ frequency response [Cassarella, Grimble] and state variable time domain modeling frameworks [Healey and Lienard, 1992, Marco, 1992], Autopilots that provide high performance (low errors over the dominant range of frequency spectrum in both regulation and tracking tasks) in spite of widely varying operating conditions are called 'robust' [Grimble]. Design techniques for robust autopilots typically use frequency response or state space techniques to specify proportional, derivative and integral control gains [Ogata]. A Sliding Mode Control can compensate for known nonlinear behavior - is convenient and has equally definable robustness properties [ Utkin, 1977, Slotine, 1986, DeCarlo, Zak, Mathews, 1988], [Healey, 1992]. This paper give (LQ) servo with command following and Sliding Mode Control which are beneficial for nonlinear systems (cancels nonlinearities), good robustness to uncertainty and practically easy to use. It attempts to show a new method in which tracking and recovery onto a moving platform is enabled. Verify the controller's performance by developing models, simulation, and experimental validation.

Section II will focus on the equations of motion for an AUV and methods for modeling AUV dynamics. Section III will discuss tracking control using conventional method and a proposed sliding control method. Section IV will present simulation and experimental results.

\section{SYSTEM DESCRIPTION}

This section develops a simplified models for diving of underwater vehicles having a 'low' drag hydrodynamic shape. It will show that for small angles of attack and side slip, linear models could be found where the system parameters were uncertain but nominally constant.

\subsection{GENERALIZED EQUATIONS OF MOTION}

This section describes the equations of motion for an AUV. It is from these equations of motion that a model can be developed for both simulation of motion as well as construction of model based controllers for AUV's. Using a Newton-Euler approach, and body-fixed reference frame as in Figure 1 and notation used in Table 1 Healey [1-4] derives the equations of motion for six degrees of freedom as:

SURGE EQUATION OF MOTION

$m\left[\dot{u}_{r}-v_{r} r+w_{r} q-x_{G}\left(q^{2}+r^{2}\right)+y_{G}(p q-\dot{r})+z_{G}(p r+\dot{q})\right]+(W-B) \sin \theta=X_{f}$

SWAY EQUATION OF MOTION

$m\left[\dot{v}_{r}+u_{r} r-w_{r} p+x_{G}(p q+\dot{r})-y_{G}\left(p^{2}+r^{2}\right)+z_{G}(q r-\dot{p})\right]-(W-B) \cos \theta \sin \phi=Y_{f}$

HEAVE EQUATION OF MOTION

$m\left[\dot{w}_{r}-u_{r} q+v_{r} p+x_{G}(p r-\dot{q})+y_{G}(q r+\dot{p})-z_{G}\left(p^{2}+q^{2}\right)\right]+(W-B) \cos \theta \cos \phi=Z_{f}$

ROLL EQUATION OF MOTION

$I_{x} \dot{p}+\left(I_{z}-I_{y}\right) q r+I_{x y}(p r-\dot{q})-I_{y z}\left(q^{2}-r^{2}\right)-I_{x z}(p q+\dot{r})+m\left[y_{G}\left(\dot{w}-u_{r} q+v_{r} p\right)\right.$

$$
\left.-z_{G}\left(\dot{v}_{r}+u_{r} r-w_{r} p\right)\right]-\left(y_{G} W-y_{B} B\right) \cos \theta \cos \phi+\left(z_{G} W-z_{B} B\right) \cos \theta \sin \phi=K_{f}
$$

PITCH EQUATION OF MOTION

$$
\begin{aligned}
& I_{y} \dot{q}+\left(I_{z}-I_{z}\right) p r-I_{x y}(q r+\dot{p})+I_{y z}(p q-\dot{r})+I_{x z}\left(p^{2}-r^{2}\right)-m\left[x_{G}\left(\dot{w}-u_{r} q+v_{r} p\right)\right. \\
& \left.-z_{G}\left(\dot{u}_{r}-v_{r} r+w_{r} q\right)\right]+\left(x_{G} W-x_{B} B\right) \cos \theta \cos \phi+\left(z_{G} W-z_{B} B\right) \sin \theta=M_{f}
\end{aligned}
$$


YAW EQUATION OF MOTION

$$
\begin{aligned}
& I_{z} \dot{r}+\left(I_{y}-I_{x}\right) p q-I_{x y}\left(p^{2}-q^{2}\right)-I_{y z}(p r+\dot{q})+I_{x z}(q r-\dot{p})+m\left[x_{G}\left(\dot{v}_{r}+u_{r} r-w_{r} p\right)\right. \\
& \left.-y_{G}\left(\dot{u}_{r}-v_{r} r+w_{r} q\right)\right]-\left(x_{G} W-x_{B} B\right) \cos \theta \sin \phi-\left(y_{G} W-y_{B} B\right) \sin \theta=N_{f}
\end{aligned}
$$

Where:

$u_{r}, v_{r}, w_{r}=$ velocities for a body fixed system with respect to the water

$p, q, r=$ angular velocities for a body fixed system

$\mathrm{W}=$ weight

$\mathrm{B}=$ buoyancy

$\mathrm{I}=$ mass moment of inertia terms

$\mathrm{x}_{\mathrm{B}}, \mathrm{y}_{\mathrm{B}}, \mathrm{z}_{\mathrm{B}}=$ position difference between geometric center of the AUV and center of buoyancy $\mathrm{x}_{\mathrm{G}}, \mathrm{y}_{\mathrm{G}}, \mathrm{z}_{\mathrm{G}}=$ position difference between geometric center of AUV and center of gravity $\mathrm{X}_{\mathrm{f}}, \mathrm{Y}_{\mathrm{f}}, \mathrm{Z}_{\mathrm{f}}, \mathrm{K}_{\mathrm{F}}, \mathrm{M}_{\mathrm{f}}, \mathrm{N}_{\mathrm{f}}=$ sums of all external forces and moments acting on an AUV in the particular body fixed direction
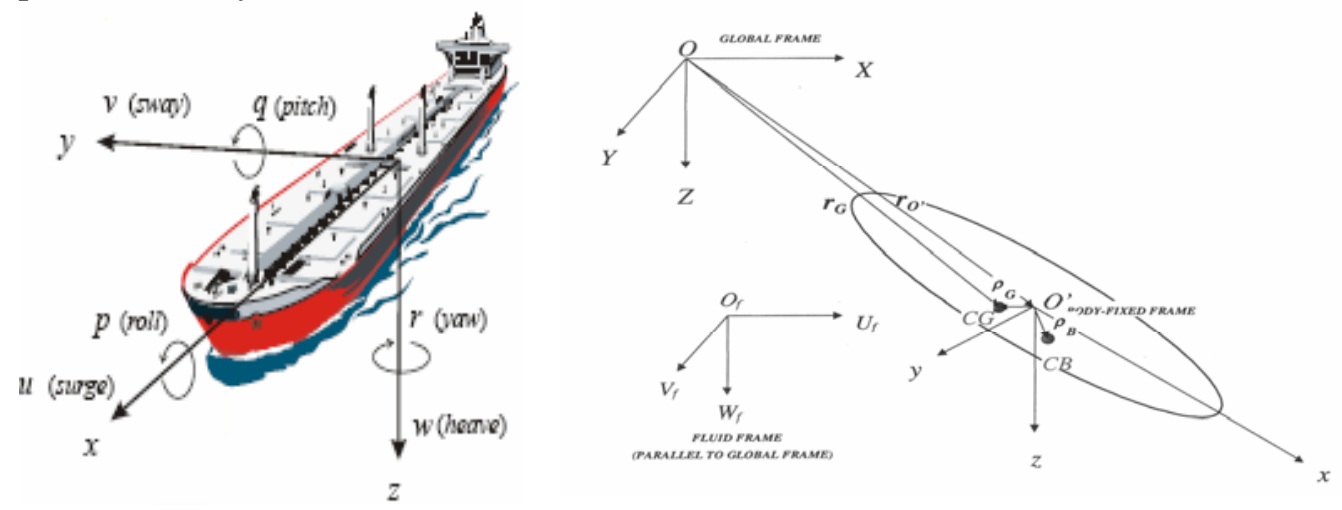

Figure 1. Body-fixed and Earth fixed reference frame

\begin{tabular}{|c|l|c|c|c|}
\hline DOF & & $\begin{array}{c}\text { Force and } \\
\text { Moments }\end{array}$ & $\begin{array}{c}\text { Linear and } \\
\text { angular velocities }\end{array}$ & $\begin{array}{c}\text { Position and } \\
\text { Euler angles }\end{array}$ \\
\hline 1 & Motion in the $x$-direction (Surge) & $\mathrm{X}$ & $u$ & $x$ \\
\hline 2 & Motion in the $y$-direction (Sway) & $\mathrm{Y}$ & $v_{r}$ & $y$ \\
\hline 3 & Motion in the $z$-direction (heave) & $\mathrm{Z}$ & $w_{r}$ & $z$ \\
\hline 4 & Rotation about the $x$-axis (Roll, heel) & $\mathrm{K}$ & $q$ & $\theta$ \\
\hline 5 & Rotation about the $y$-axis (pitch, trim) & $\mathrm{M}$ & $r$ & $\psi$ \\
\hline 6 & Rotation about the $z$-axis (yaw) & $\mathrm{N}$ & $r$ & \\
\hline
\end{tabular}

Table 1

\subsection{Diving Equation of Motion}

A diving system model will be developed from the above equations of motion. The primary variables of interest are $w_{r}, q, \theta$ and $\mathrm{z}$ while $v_{r}, \mathrm{r}, \mathrm{p}, \varphi, \psi, x, y$ are neglected. Assuming the vehicle is already in forward motion, under constant forward speed relative to the water, all products of small motions are ignored and the horizontal plane motions coupled to the vertical plane equations can be dropped. Primarily considering the effects of vehicle inertia, hydrostatic and weight terms, and hydrodynamic force components from lift and added mass a set of simplified equations of motion are developed To handle the force and moment terms, an assumption of "small" motions is made to develop "hydrodynamic coefficients" that can be defined relative to the individual motion components [3-4]. This will allow the description of the forces and moments as a function of vehicle dynamic states.

For heave motion, equation (3), the force in the $\mathrm{z}$ direction is:

$Z_{f}=Z_{\dot{w}_{r}} \dot{w}_{r}+Z_{w_{r}} w_{r}+Z_{\dot{q}} \dot{q}+Z_{q} q$

and for pitch motion, equation (4), the rotational moment is: 
$M_{f}=M_{\dot{w}} \dot{w}_{r}+M_{w_{r}} w_{r}+M_{\dot{q}} \dot{q}+M_{q} q$

This leads to: $Z_{\dot{w}_{r}}=\frac{\partial Z_{f}}{\partial \dot{w}_{r}} ; Z_{w_{\mathrm{r}}}=\frac{\partial Z_{\mathrm{f}}}{\partial w_{\mathrm{r}}} ; Z_{\dot{\mathrm{q}}}=\frac{\partial Z_{\mathrm{f}}}{\partial \dot{q}} ; Z_{q}=\frac{\partial Z_{\mathrm{f}}}{\partial q}$;

and $\quad M_{\dot{w}_{\mathrm{r}}}=\frac{\partial M_{\mathrm{f}}}{\partial \dot{w}_{\mathrm{r}}} ; M_{w_{\mathrm{r}}}=\frac{\partial M_{\mathrm{f}}}{\partial w_{\mathrm{r}}} ; M_{\dot{\mathrm{q}}}=\frac{\partial M_{\mathrm{f}}}{\partial \dot{q}} ; M_{\mathrm{q}}=\frac{\partial M_{\mathrm{f}}}{\partial q}$;

Where:

$Z_{\dot{w}_{r}}=$ added mass due to heave velocity

$M_{\dot{q}}=$ added mass due to pitch rate

$Z_{w_{r}}=$ coefficient of heave force induced by heave velocity

$Z_{q}=$ coefficient of heave force induced by pitch rate

$M_{\dot{w}_{r}}=$ added mass moment of inertia due to heave velocity

$M_{\dot{q}}=$ added mass moment of inertia due to pitch rate

$M_{w_{r}}=$ coefficient of pitch moment induced by heave velocity

$M_{q}=$ coefficient of pitch moment induced by pitch rate

In addition, the action of the planes will produce forces that when linearized are $Z_{\delta_{\mathrm{pl}}} \delta_{p l}(\mathrm{t})$ and $M_{\delta_{\mathrm{pl}}} \delta_{p l}(\mathrm{t})$. The dynamics of the vehicle are thus defined as:

$$
\begin{aligned}
& u_{r}=U_{0} \\
& m \dot{w}_{r}=m U_{0} q+(W-B) \cos \theta+Z_{\dot{w}_{r}} \dot{w}_{r}+Z_{w_{r}} w_{r}+Z_{\dot{q}} \dot{q}+Z_{q} q+Z_{\delta_{p l}} \delta_{p l}(t) \\
& I_{y y} \dot{q}=\left(z_{B} B-z_{G} W\right) \sin \theta+M_{\dot{q}} \dot{q}+M_{q} q+M_{\dot{w}_{r}} \dot{w}_{r}+M_{w_{r}} w_{r}+\mathrm{M}_{\delta} \delta_{p l} \\
& \dot{\theta}=q \\
& \dot{Z}=w_{r} \cos \theta-U_{0} \sin \theta
\end{aligned}
$$

Further assumptions of bow and stern planes operate equal and opposite, small pitch angle, and small motions in the vertical plane [4], therefore $\mathrm{Z}_{\delta}=0, \sin \theta \approx \theta$ and $\cos \theta \approx 1$, results in a set of linerized equations that can be put in the in matrix form,

$M M \dot{\mathbf{x}}=\boldsymbol{A A} \mathbf{x}+\boldsymbol{B B} u$ :

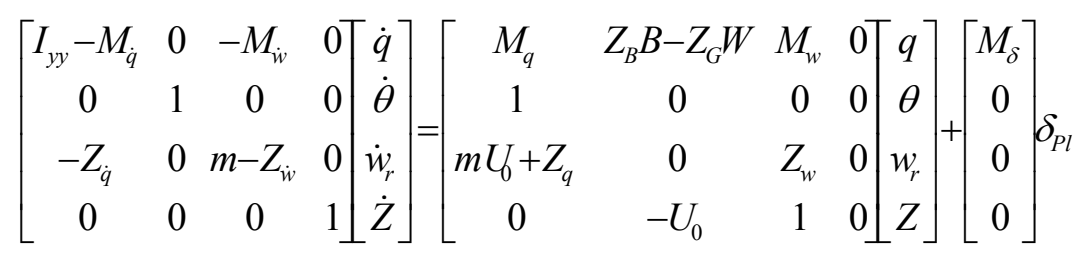

Equation (14) can be rewritten in the form $\dot{\mathbf{x}}=\mathbf{A x}+\mathbf{B} u$ where $\mathbf{A}=\boldsymbol{M} \boldsymbol{M}^{-1} \boldsymbol{A} \boldsymbol{A}$ and $\mathbf{B}=\boldsymbol{M} \boldsymbol{M}^{-1} \boldsymbol{B} \boldsymbol{B}$ and $\boldsymbol{u}=\boldsymbol{\delta}_{p l}$

\section{Sliding Mode Control}

The most highly developed aspect of Sliding Mode Control is with a nonlinear system [5-8]. The control law is composed of a nonlinear cancellation term, a linear dynamics substitution term, and a switching term for robustness. A similar control law may be found for a system model that is predominantly linear where the control again contains a cancellation term, a substitution term, and a switching term. The robustness achieved by the switching term also 
applies. As an application of Lyapunov method, consider a single input system linear in control effort.

$$
\dot{\mathbf{x}}=f(x)+g(x) u
$$

Where $f(\mathrm{x}), g(\mathrm{x})$ are in general, nonlinear functions in $x$. We want to design $\boldsymbol{u}$ such that we guarantee stability of $x=0$.

Choose the Lyapunov function

$$
v(x)=\frac{1}{2}[\sigma(x)]^{2}, \text { where } \sigma(x)=s^{T} x
$$

The scalar function $\sigma(x)$ can viewed as a weighted sum of the errors in the states $x$.

For stability, we want the time derivative of $v(x)$ to be negative, i.e $\dot{v}(x)=[\sigma(x) \dot{\sigma}(x)]<0$

Which can achieved if $\sigma(x) \dot{\sigma}(x)=-\eta^{2} \mid \sigma$, which mean that $\dot{\sigma}(x)=-\eta^{2} \operatorname{sign}(\sigma)$

Using $\sigma(x)=s^{T} x$, we get $\dot{\sigma}(x)=s^{T} \dot{x}=s^{T} f(x)+s^{T} g(x) u=-\eta^{2} \operatorname{sign}(\sigma)$,

Solving for $u$ we get the control law

$$
u=-\left[s^{T} g(x)\right]^{-1} s^{T} f(x)-\left[s^{T} g(x)\right]^{-1} \eta^{2} \operatorname{sign}(\sigma)
$$

We can see that this control law is the sum of two terms. The first term is nonlinear state feedback, and the second term is a switching control law. The term $\eta^{2}$ is an arbitrary positive quantity, we usually select it such that $v$ is negative even in the presence of modeling errors and disturbances. The above control law guarantee stability of $\sigma(x)=0$, or $\sigma^{\mathrm{T}} x=0$. We need to find $(S)$ such that stability of $x=0$ is guaranteed. If $\sigma(x)=0$, the system becomes $u=-\left[s^{T} g(x)\right]^{-1} s^{T} f(x)$, and

$$
\dot{\mathbf{x}}=f(x)-g(x)\left[s^{T} g(x)\right]^{-1} s^{T} f(x) ;
$$

If we linearize this system, we get,

$\dot{\mathbf{x}}=\mathbf{A x}+\mathbf{B u} ;$ Where $\boldsymbol{x} \in \mathfrak{R}^{n^{* 1}} ; \boldsymbol{A} \in \mathfrak{R}^{n^{* n}} ; \boldsymbol{B} \in \mathfrak{R}^{n^{*} r} ; \boldsymbol{u} \in \mathfrak{R}^{r^{* 1}}$ and $A=\left.\frac{\partial f}{\partial x}\right|_{0}, B=g(0)$

Then, on $\sigma(x)=0$ we have $\dot{\mathbf{x}}=\mathbf{A} x-B\left(s^{T} B\right)^{-1} S^{T} \mathbf{A} x=\left[\mathbf{A}-B\left(s^{T} B\right)^{-1} S^{T} \mathbf{A}\right\rfloor x$

This means that the closed loop dynamics matrix is

$$
\begin{gathered}
A_{C}=A-B\left(s^{T} B\right)^{-1} S^{T} \mathbf{A}=A-B k, \text { i.e } k=\left(s^{T} B\right)^{-1} S^{T} \mathbf{A} \text { or } \quad S^{T} \mathbf{A}-S^{T} B k=0 \\
\text { Or } S^{T}(\mathbf{A}-B k)=0 \text { i.e }(\mathbf{A}-B k)^{T} S=0 \text { or } A_{c}^{T} S=0
\end{gathered}
$$

From equation (21), $S$ is the eigenvector of the closed loop dynamic matrix corresponds to zero eigenvalue.

\section{Simulation Results}

The simplified dynamics model in equation(14), may be employed and the kinematic relationships may be linearized for shallow dive angle where $\boldsymbol{x}^{\prime}=\left[w_{r}, q, \theta, Z\right]$ is the state vector; and $u=\delta_{p l}(t)$ is the input. Numerical values for the hydrodynamic coefficient $[4,9]$ are given as follows:

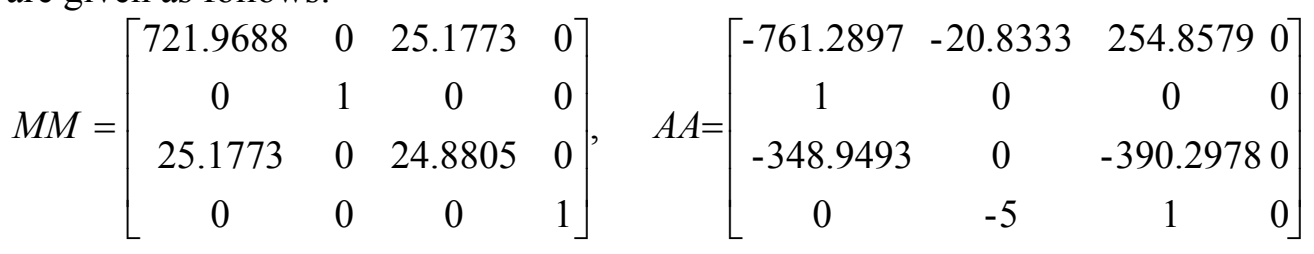




\subsection{Open loop system analysis}

The plant has 4 poles $(0,-0.0214,-1.3879$ and -15.8077$)$ and 2 zeros $(-6.1054$ and12.6951). This means that it contains a natural integrator in addition to sluggish and undesirable response. A popular modern approach to the design of robust controllers is linear quadratic regulator. This approach has been used extensively in the design. The optimal control law which minimizes the following quadratic cost functions $J(u)=\int_{0}^{\infty}\left(y^{2}+\rho u^{2}\right) d t$ where $\rho$ is the control weighting parameter.

With $\rho=400$, the optimal state feedback gains $\mathrm{G}=\left[\begin{array}{llll}-0.7250 & -1.0930 & -0.0371 & 0.0500\end{array}\right]$ and $u=-G x$, As expected from LQR theory $[8,10]$, the feedback loop has nice properties when examined at the plant input; i.e. when the loop is broken at the plant input with $\mathrm{G}_{\mathrm{LQ}}=\mathrm{G}(\mathrm{SI}-\mathrm{A})^{-1} \mathrm{~B}$.

\subsection{Simulation Results for LQ Servo with Command Following}

Now suppose that LQ design is rearranged to accommodate reference commands. To do this, we wish to replace the following closed loop dynamical system

$\dot{\mathbf{x}}=A x+B u=A x-B G x=A x-B\left[\begin{array}{llll}g_{1} & g_{2} & g_{3} & 0\end{array}\right] x-B g_{4} y$

With the following:

$\dot{\mathbf{x}}=A x+B u=A x-B G x=A x-B\left[\begin{array}{llll}g_{1} & g_{2} & g_{3} & 0\end{array}\right] x-B g_{4}(\mathrm{r}-\mathrm{y})$

Here, represent a reference command that has been introduced in the appropriate place. The error signal $e=r-y$ is the so-called tracking error.

Finally, the ten meters step response of the closed loop system for the output (z) is shown in Figure 2, the response has good time domain specifications with a ten percentage overshoot and 18 second settling time but it needs more effort to compensate.

\subsection{Simulation Results for Sliding Mode Control}

In development of Sliding Mode Controller, a sliding surface is created from a linear combination of the state variable errors, ignoring any nonzero pitch angle and rate commands: $\sigma(t)=s^{\prime}\left(\mathbf{x}-\mathbf{x}_{c o m}\right)$

Now pole placement is utilized to obtain linear state feedback gains $\mathbf{k}$, with at least one of the poles placed at zero. The closed loop dynamics matrix can then be calculated where $\mathbf{A}_{\mathbf{c}}=\mathbf{A}-\mathbf{B k}$ and the sliding surface polynomial $(S)$ is found from the left eigenvector of $\mathbf{A}_{\mathbf{c}}^{\prime} s=0$. The resulting control law is then obtained from:

the MATLAB command for pole placement that gives the linear state feedback gain $\mathbf{k}=\operatorname{place}(\mathbf{A}, \mathbf{B},[-1.2+1.2 \mathrm{i},-1.2-1.2 \mathrm{i},-1.2,0])$ which gives

$\mathbf{k}=[-138.6,-0.2807,-156.9654,0]$; as the feedback gain set to place the closed loop poles when in the sliding condition at $\lambda=[-1.2+1.2 \mathrm{i},-1.2-1.2 \mathrm{i},-1.2,0]$. One pole is required to be at the origin to allow for the single sliding constraint for the single input as mentioned in the discussion earlier. The closed loop dynamics matrix is $\mathbf{A}_{\mathbf{c}}$ and the eigenvector of $\mathbf{A}^{\prime}{ }_{\mathrm{c}}$ for the pole at the origin is the sliding surface $S=\left[\begin{array}{llll}0.6531 & -0.0353 & 0.7564 & 0.0050\end{array}\right]$, and $u=-\left[s^{T} g(x)\right]^{-1} s^{T} f(x)$.

The current depth controller is designed primarily to act as a depth regulator, where once a command for depth is received; its job is to approach the commanded depth with desired characteristics and stability. Figure 3 shows the response of AUV depth controller to a ten meter change in commanded depth. This response is excellent when the mode of the depth controller is acting as regulator, responding to step changes in depth but It can be seen there is 
a significant phase lag in the depth achieved. A sliding mode control could be developed and tuned to better handle this deficiency. Figure 4 shows error vs. change of error curve for LQ and SMC controllers. It prove no chattering or oscillation for both controllers.

\subsection{Robustness}

As with the many other control laws this method (SMC) relies heavily on the knowledge of the model of the signal being tracked as well as a model of the system being controlled. This modeling requirement can be a limitation for the controller depending on the ability to accurately model both systems. This particular design however, is robust in that it can handle errors in modeling the dynamics of the input signal that is being tracked.

As a test of the robustness, add a random noise to states, performance of the controller was examined when errors in modeling the reference signal were present. For this case, once the gains for the controller were chosen based on an assumed model of the reference signal, the controller was able to track a signal of different set of model parameters with little degradation in tracking performance as shown in Figure (5). Robustness analysis during tracking of exogenous inputs is essentially unchanged from the above analysis because robustness is achieved primarily through the feedback of measured response

\section{CONCLUSION}

This paper has shown the utilization of a model based, LQ-servo command following, and slide mode controllers for an AUV tracking with a time varying depth command. The LQ-servo command following Controller has proven to be fairly robust and readily tunable. However, there are limitations, in particular its sensitivity to errors in the input signal. In addition during implantation, the entire state must be measured. To follow a desired trajectory that forms the basis of many guidance requirements, a sliding mode controller (SMC) is presented that has been experimentally validated under a wide variety of conditions. The SMC is more beneficial for nonlinear systems, good robustness to uncertainty and it is practically easy to use. This controller design provides a means by which an AUV could efficiently compensate for the time varying depth.

\section{References}

[1] Fossen, T. I. (1994). “Guidance and Control of Ocean Vehicles”, John Wiley \& Sons Ltd. ISBN 0-471-94113-1

[2] Michael S. Triantafyllou and Franz S. Hover Maneuvering and Control of Marine Vehicles MIT, 2003

[3] Healey, A.J., Dynamics of Marine Vehicles (ME-4823), Class Notes, Naval Postgraduate School, Monterey, CA, 1995.

[4] Marco, D.B. and A.J. Healey, "Current Developments in Underwater Vehicle Control and Navigation," Proceedings of IEEE Oceans, 2000.

[5] Ramirez, Herbertt,"A Dynamical Variable Structure Control Strategy in Asymptotic Output Tracking Pproblems", IEEE Transactions on Automatic Control, Vol.38, No. 4, April 1993

[6] Lienard, D. and A.J. Healey, "Multivariable Sliding Mode Control for Autonomous Diving and Steering of Unmanned Underwater Vehicles, Journal of Oceanic Engineering, vol. 18, pp 327-339, July 1993.

[7] Lienard, David, "Autopilot Design for Autonomous Underwater Vehicles Based on Sliding Mode Control,” M.S. Thesis, Naval Postgraduate School, Monterey, CA, June 1990 
[8] Gelb, A. ed. "Applied Optimal Estimation" MIT Press 10th printing 1988 ISBN \# 0-262-57048-3.

[9] Healey, A. J., Lienard, D., "Multivariable Sliding Mode Control for Autonomous Diving and Steering of Unmanned Underwater Vehicles", IEEE Journal of

Oceanic Engineering Vol. 18, No. 3, July 1993 pp. 1-13

[10] Franklin G.F, Powell, Emami-Naeini. Feedback Control of Dynamic Systems. $4^{\text {th }}$ Edition New Jersey:Prentice Hall 2002.

[11] DeCarlo, R.A., Zak, S. H., Mathews, G. P., "Variable Structure Control of NonLinear Multivariable Systems: A Tutorial", Proc IEE, Vol 76, 1988.

Heave(rad/sec), pitch rate(rad/sec), pitch angle(deg.), depth $(\mathrm{m})$ and planes angle(deg) vs time
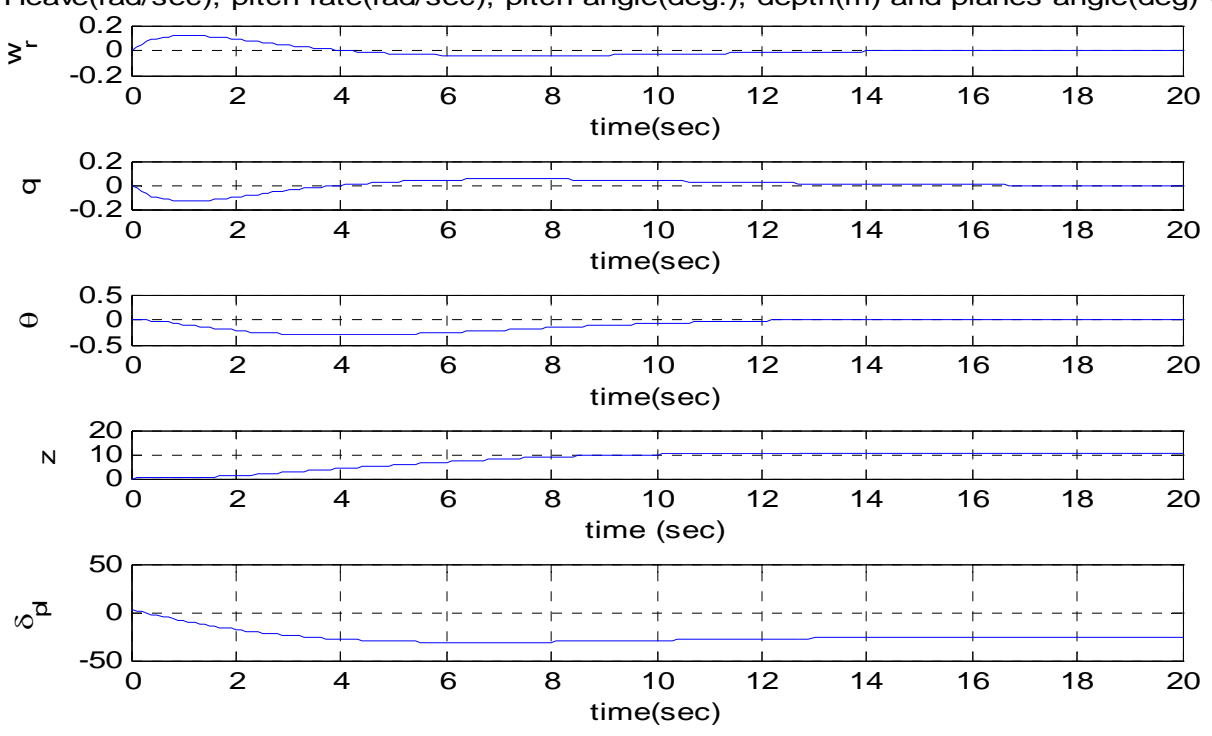

Figure 2: LQ Servo with Step Command Following
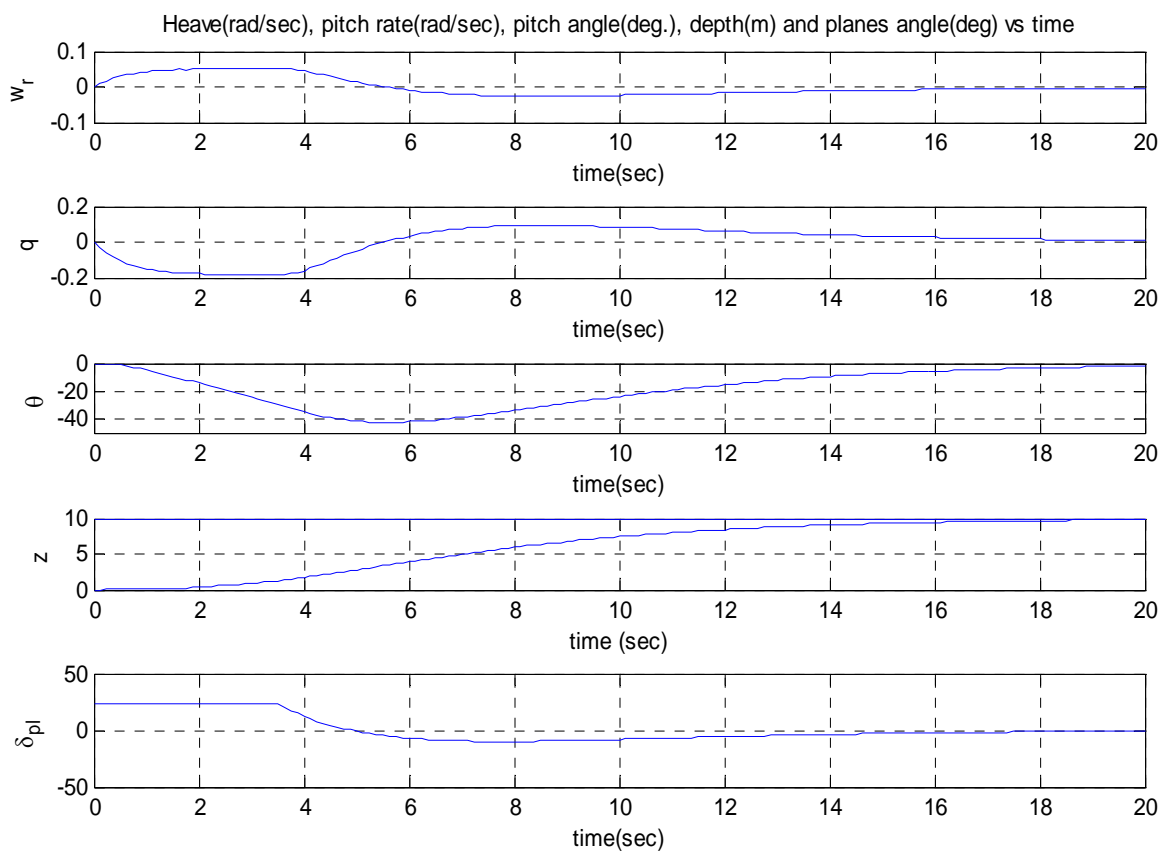

Figure 3: Sliding Mode Controller 

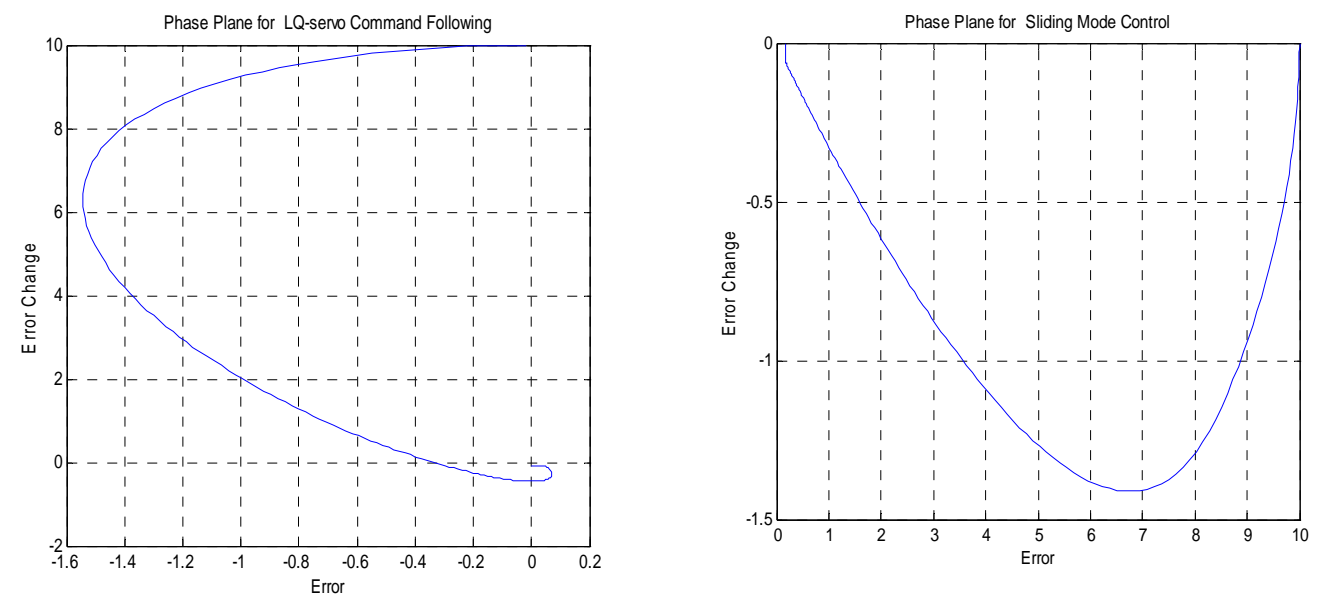

Figure 4: Error vs. Change of Error curve for LQ and SMC controllers

Heave(rad/sec), pitch rate(rad/sec), pitch angle(deg.), depth(m) and planes angle(deg) vs time
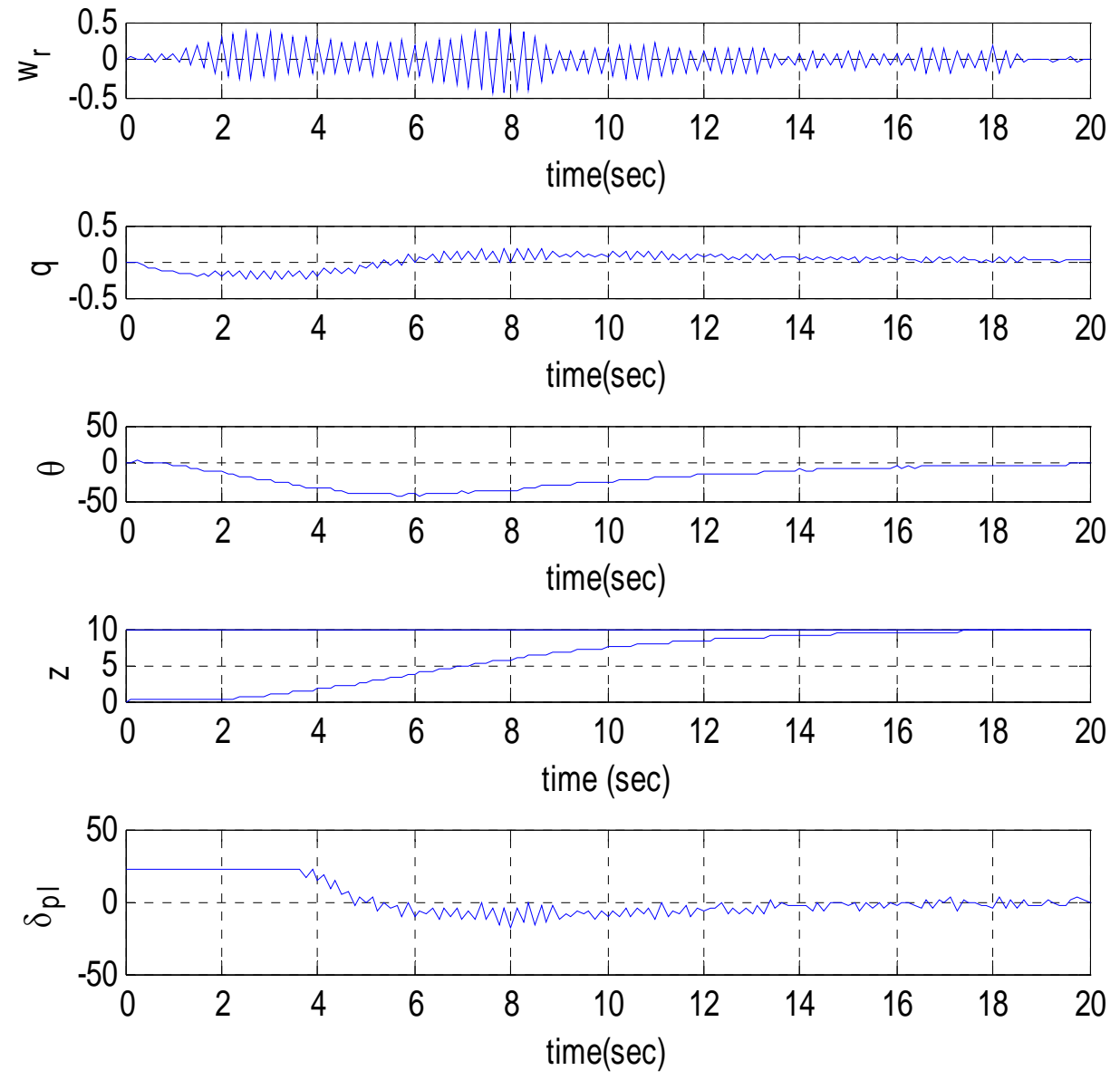

Figure 5: Sliding Mode Controller with random noise in states 